\title{
Functional and Structural Arterial Vessel Features of Female Patients with Stable Dermatomyositis and Antisynthetase Syndrome
}

\author{
Rafael Giovani Misse1, Isabela Bruna Pires Borges1, Valéria Aparecida Costa Hong2, \\ Luiz Aparecido Bortolotto², Samuel Katsuyuki Shinjo ${ }^{1^{*}}$ (1)
}

\author{
${ }^{1}$ Division of Rheumatology, Faculdade de Medicina FMUSP, Universidade de Sao Paulo, Sao Paulo, SP, Brazil (BR) \\ ${ }^{2}$ Hypertension Unit, Instituto do Coração, Hospital das Clinicas HCFMUSP, Faculdade de Medicina, Universidade de Sao Paulo, \\ Sao Paulo, SP, Brazil (BR) \\ Email: *samuel.shinjo@gmail.com
}

How to cite this paper: Misse, R.G., Borges, I.B.P., Hong, V.A.C., Bortolotto, L.A. and Shinjo, S.K. (2019) Functional and Structural Arterial Vessel Features of Female Patients with Stable Dermatomyositis and Antisynthetase Syndrome. Open Journal of Rheumatology and Autoimmune Diseases, 9, 101-110.

https://doi.org/10.4236/ojra.2019.93009

Received: August 7, 2019

Accepted: August 25, 2019

Published: August 28, 2019

Copyright $\odot 2019$ by author(s) and Scientific Research Publishing Inc. This work is licensed under the Creative Commons Attribution International License (CC BY 4.0).

http://creativecommons.org/licenses/by/4.0/ (c) (i) Open Access

\begin{abstract}
Introduction: Dermatomyositis (DM) and antisynthetase syndrome (ASS) show a high frequency of metabolic syndrome, which can be preceded by endothelial dysfunction and arterial stiffness. To date, only one study has evaluated these vessel parameters in DM, and no study of ASS exists. Therefore, the aim of the study was to assess the structural and functional arterial of arterial vessels in DM and ASS. Methods: This cross-sectional study enrolled 21 adult female patients (14 DM and 7 ASS) who were age-, gender- and ethnicity-matched to 12 healthy individuals. Patients using lipid lowering agents or prednisone at doses $\geq 0.25 \mathrm{mg} / \mathrm{kg} / \mathrm{day}$, and patients with uncontrolled systemic arterial hypertension, diabetes mellitus, cardiac insufficiency, and disease activity were excluded. Arterial stiffness was evaluated using carotid-femoral pulse wave velocity (PWV), and endothelial function was evaluated using dependent flow-mediated dilatation (FMD) of the brachial artery. Results: The mean age of patients with DM or ASS were $45.4 \pm 8.6$ and $44.0 \pm 6.1$ years, respectively $(P=1.000)$, and patients were predominantly of white ethnicity. Six DM patients and three ASS patients had systemic arterial hypertension, whereas $9 \mathrm{DM}$ patients and six ASS patients had dyslipidemia. Endothelial baseline diameter, hyperemia diameter and FMD values were similar among the three groups $(P>0.05)$. Moreover, the median FMD values were also similar between the patients with DM and patients with ASS [8.3\% $(4.5 \%-10.9 \%)$ vs. $6.0 \%(-1.8 \%-8.2 \%) ; P=0.585]$. The PWV values were comparable among the three groups $(P=0.253)$. In addition, no difference was observed between patients with DM and patients with ASS $(7.4 \pm 0.8 \mathrm{~m} / \mathrm{s}$ vs. $7.4 \pm 0.9 \mathrm{~m} / \mathrm{s} ; P=1.000)$. Conclusions: Despite the high prevalence of dyslipidemia and systemic arterial hypertension, our female patients with sta-
\end{abstract}


ble DM and ASS had FMD and PWV values comparable to those of the control group.

\section{Keywords}

Arterial Stiffness, Atherosclerosis, Endothelial Dysfunction, Systemic Autoimmune Myopathies

\section{Introduction}

Dermatomyositis (DM) and antisynthetase syndrome (ASS) are part of a group of rare diseases called systemic autoimmune myopathies (SAMs). The main clinical symptoms of these diseases are progressive, symmetrical and predominantly proximal limb weakness [1] [2]. DM typically includes cutaneous lesions, such as heliotrope rash, Gottron's signal and papules [1] [2]. In ASS, fever, "mechanic's hands", Raynaud's phenomenon, and pulmonary and joint involvements may occur [3].

Several studies have demonstrated high cardiovascular abnormalities (i.e., pressure dysfunction, heart rate variability and reactive brachial artery abnormality) [4] [5] [6] and metabolic syndrome in SAMs [7] [8] [9] [10]. These factors may contribute to the development and progression of atherosclerosis, resulting in considerable morbidity and mortality in patients with cardiovascular diseases [4] [5] [6].

Physiopathologically, atherosclerosis is characterized by early endothelial dysfunction and increased arterial stiffness [4] [5]. In this context, flow-mediated vasodilatation (FMD) and pulse wave velocity (PWV) assessments have been used extensively in clinical practice as non-invasive, validated and reproducible techniques for subclinical atherosclerosis assessment [4] [5].

In the only available study in the literature, Vincze et al. [7] demonstrated the impairment of dependent FMD and increased arterial stiffness in patients with DM. However, the authors did not provide details about the patients' age, gender, clinical and laboratory features or treatment. Because these parameters can interfere with FMD and arterial stiffness values, this study's results should be analyzed with caution. Finally, to the best our knowledge, no study has assessed FMD and PWV in patients with ASS.

Therefore, the goal of the present study was to assess the structural and functional behavior of arterial vessels in patients with stable forms of DM or ASS, as compared with age-, gender- and ethnicity-matched to healthy individuals.

\section{Subject and Methods}

Study design. Between 2017 and 2018, a cross-sectional single-center study was conducted at Hospital das Clinicas, Faculdade de Medicina da Universidade de São Paulo (Brazil), in which 91 consecutive adult patients with defined systemic autoimmune myopathies (60 DM patients and 31 ASS patients) under regular 
follow-up observation were initially evaluated. This study was approved by the local Ethics Committee (CAPPesq, number 2.881.819) and all participants signed the informed consent form.

\section{Patients}

Patients with DM fulfilled the European League against Rheumatism/American College of Rheumatology (EULAR/ACR 2017) classification criteria for SAMs [11]. Definition of ASS was based on the study of Connors et al. [3].

\section{Exclusion Criteria}

Patients with clinical and laboratory disease activity, overlapped syndromes, diabetes mellitus, uncontrolled systemic arterial hypertension, or cardiac insufficiency were excluded, as were patients using lipid-lowering agent or prednisone at doses of $\geq 0.25 \mathrm{mg} / \mathrm{kg} /$ day in the last 3 consecutive months.

\section{Patients' Data}

The following data were obtained from the patients:

1) Demographic data: current age, gender and body mass index (BMI) $\left(\mathrm{kg} / \mathrm{m}^{2}\right)$.

2) Laboratory data: creatine phosphokinase ( $\mathrm{CPK}$ ), aspartate aminotransferase (AST), alanine aminotransferase (ALT), lactic dehydrogenase (LDH), analyzed using automated kinetic method.

3) Disease parameters: disease duration and current disease status assessed using the International Myositis Assessment \& Clinical Studies Group (IMACS) set scores: Manual Muscle Testing (MMT)-8, Myositis Disease Activity Assessment Visual Analogue Scales (MYOACT), global assessment of disease by physician and patient through the Visual Analogue Scales (VAS), Health Assessment Questionnaire (HAQ), and serum levels of the muscle enzymes [12]-[17].

4) Treatment: current treatments, including glucocorticoid and immunosuppressive drugs.

5) Comorbidities: systemic arterial hypertension and dyslipidemia: total cholesterol $>200 \mathrm{mg} / \mathrm{dL}$, high density lipoprotein (HDL)-cholesterol $<40 \mathrm{mg} / \mathrm{dL}$ and/or low density lipoprotein (LDL)-cholesterol $>130 \mathrm{mg} / \mathrm{dL}$ [18].

6) Endothelial function assessment: The test was performed in the morning, after the patients had fasted for 8 hours. They were tested and on the right arm. The patients were instructed to remain fasting and without vasoactive medications for $12 \mathrm{~h}$ before evaluation. The environment of the examination was silent, with low luminosity and maintained temperature between $20^{\circ} \mathrm{C}$ and $25^{\circ} \mathrm{C}$. Images of the right brachial artery located $7 \mathrm{~cm}$ above the medial humeral epicondyle were recorded for measurements of blood flow velocity and arterial resting diameter. For FMD (endothelium-dependent) register, a sphygmomanometer was placed on the forearm and inflated to a pressure of at least $50 \mathrm{mmHg}$ above systemic pressure for $5 \mathrm{~min}$, inducing reactive hyperemia maneuver. Images were captured during $3 \mathrm{~min}$ after cuff release. The diameter of the artery was through ultrasound equipment (Sequoia Echocardiography System $^{\circledR}$, version 6.0, Siemens, CA, USA). The different phase of the examination was recorded on computer for later analysis. FMD\% (endothelium-dependent) was calculated according to the formula below: FMD\% = Diameter after hyperemia - Resting 
diameter $\times 100 /$ Resting diameter. Moreover, four healthy subjects were recruited to measure inter-observer reproducibility, which were evaluated at different times of the day by the same evaluator.

7) Aortic stiffness assessment: Before the evaluation, the patients performed 5 minutes of rest in dorsal decubitus in silence. Later, their systolic blood pressure (SBP) and the diastolic blood pressure (DBP) values were assessed using automatic non-invasive PWV (m/s) measurements by the Complior ${ }^{\circledR}$ device (Colson, Gargesles Gonesses, France). Two typing scanners (TY 306, Tokyo, Japan) were positioned simultaneously in the carotid and femoral arteries, located at a previously measured distance. These sensors contain membranes which are successively deformed by the shock of the pulse wave, and this deformation is initially transformed into an electrical signal and transmitted to a computerized calculation program.

PWV was analyzed by means of the carotid-femoral trajectory. Each pulsating wave appears real time on the computer screen, and the apparatus determines through the tangent to the initial ascending phase of the pulse wave, the beginning of the wave at the two locations and deduces, as a function of the distance between the two points, measured with tape measure, the pulse wave velocity, i.e., $\mathrm{PWV}=\mathrm{L} / \mathrm{dL}$ to the time delay between the pulse waves. To obtain the PWV value, 10 curves were considered and the mean was calculated. The curves were acquired with the patients in horizontal dorsal decubitus and analyzed by an experienced and independent evaluator.

The control (CTR) group was composed of employees from our tertiary center, who were age-, gender- and ethnicity-matched to patients with DM and patients with ASS.

\section{Statistical Analysis}

The normality of the data was verified by Shapiro Wilk adherence test with described level of $\alpha=0.5$. ANOVA or Kruskal Wallis test was utilized for comparison between DM, ASS and CTR group. Whereas, Student's t-test or Mann Whitney test were used for comparison between independent means between the DM and ASS groups. To verify the reproducibility of FMD measurement the intra-class correlation coefficient was used. Fisher's exact test and chi-square test were used for the categorical variables of two independent groups. $P$ value $\leq 0.05$ was considered statistically significant. The software used was the SPSS 22 (Chicago, IL, USA).

\section{Results}

Forty-four out of 91 patients fulfilled the present study's inclusion criteria. However, 23 patients refused to participate, due to personal motivations, work schedules, resulting in 21 female participants: 14 DM patients and 7 ASS patients. These patients were age-, gender- and ethnicity-matched to 12 individuals from the CTR group.

The patients and CTR group had a comparable mean age: DM $(45.4 \pm 8.6$ years), ASS (44.0 \pm 6.1 years) and CTR $(43.9 \pm 7.2$ years), and were predomi- 
nantly of white ethnicity. The mean disease duration was 6.0 years (DM: $5.3 \pm 3.5$ years and ASS: $6.1 \pm 2.8$ years), as shown in Table 1. BMI varied among patients

Table 1. General features of patients and control group.

\begin{tabular}{ccccc}
\hline & DM $(\mathrm{n}=14)$ & ASS $(\mathrm{n}=7)$ & CTR $(\mathrm{n}=12)$ & $P$ value \\
\hline Age (years) & $45.4 \pm 8.6$ & $44.0 \pm 6.1$ & $43.9 \pm 7.2$ & 0.304 \\
Disease durations (years) & $5.3 \pm 3.5$ & $6.1 \pm 2.8$ & - & - \\
BMI $\left(\mathrm{kg} / \mathrm{m}^{2}\right)$ & $29.9 \pm 7.4$ & $33.2 \pm 4.8$ & $25.9 \pm 6.6$ & 0.039 \\
Female gender & $14(100)$ & $7(100)$ & $14(100)$ & $>0.999$ \\
White ethnicity & $11(78.5)$ & $6(85.6)$ & $10(83.3)$ & 0.621 \\
\hline
\end{tabular}

Data are expressed as mean \pm standard deviation, or frequency (\%). ASS: antisynthetase syndrome; BMI: body mass index; CTR: control; DM: dermatomyositis.

Table 2. Disease status and treatment of the patients with dermatomyositis and antisynthetase syndrome.

\begin{tabular}{|c|c|c|c|}
\hline & $\mathrm{DM}(\mathrm{n}=14)$ & ASS $(n=7)$ & $P$ value \\
\hline MMT-8 & $80(80-80)$ & $80(80-80)$ & $>0.999$ \\
\hline MYOACT (0 - 60) & $0.0(0.0-0.0)$ & $0.0(0.0-0.0)$ & 0.336 \\
\hline Physician's VAS $(0-10 \mathrm{~cm})$ & $0.0(0.0-0.0)$ & $0.0(0.0-0.0)$ & $>0.999$ \\
\hline Patient's VAS $(0-10 \mathrm{~cm})$ & $0.0(0.0-0.2)$ & $2.5(2.4-4.2)$ & 0.155 \\
\hline HAQ $(0.00-3.00)$ & $0.00(0.00-0.20)$ & $0.5(0.0-1.00)$ & 0.289 \\
\hline CPK (U/L) & $107(91-180)$ & $162(92-253)$ & 0.287 \\
\hline AST (U/L) & $10(11-18)$ & $13(11-28)$ & 0.689 \\
\hline $\operatorname{ALT}(\mathrm{U} / \mathrm{L})$ & $12(11-23)$ & $12(20-28)$ & 0.799 \\
\hline $\mathrm{LDH}(\mathrm{U} / \mathrm{L})$ & $202(170-209)$ & $210(210-304)$ & 0.335 \\
\hline \multicolumn{4}{|l|}{ Prednisone } \\
\hline Current use & $4(28.4)$ & 0 & - \\
\hline Current dose (mg/day) & $2.5(0.0-5.0)$ & - & - \\
\hline IS/IM/Immunobiological & $12(85.5)$ & $5(71.4)$ & - \\
\hline Azatioprine & $5(35.7)$ & $2(28.5)$ & 0.799 \\
\hline Methotrexate & $2(14.2)$ & $1(14.2)$ & 0.636 \\
\hline Mycophenolate of mofetil & $3(21.4)$ & $2(28.5)$ & 0.799 \\
\hline Lefunomide & $1(7.1)$ & 0 & - \\
\hline Rituximab & $1(7.1)$ & 0 & - \\
\hline Other drugs & $6(42.8)$ & $3(42.8)$ & $>0.999$ \\
\hline Beta blocker & $3(21.4)$ & 0 & - \\
\hline Calcium channel blocker & $3(21.4)$ & $3(42.8)$ & 0.630 \\
\hline
\end{tabular}

Data are expressed as mean \pm standard deviation; median (interquartile 25 th -75 th) or frequency (\%). ALT: alanine aminotransferase; ASS: antisynthetase syndrome; AST: aspartate aminotransferase; CTR: control; CPK: creatine phosphokinase; DM: dermatomyositis; HAQ: Health Assessment Questionnaire; IM: immunomodulatory; IS: immunosuppressive; LDH: lactate dehydrogenase; MMT8: Manual Muscle Testing; MYOACT: Myositis Disease Activity Assessment; VAS: Visual Analogue Scale. 
with ASS and patients with DM, as well as in the CTR group $(P=0.039)$. However, patients with ASS and patients with DM had similar BMIs $(P=0.617)$.

Patients with ASS and patients with DM had stable diseases (Table 2) according to the IMACS set scores. Moreover, only 4 patients with DM used prednisone, and the median dose was $2.5 \mathrm{mg}$ /day. No ASS patients used glucocorticoids. In general, $85.5 \%$ of patients with DM and $71.4 \%$ of patients with ASS used at least one immunosuppressive, immunomodulatory or immnobiological drug (azathioprine, methotrexate, mycophenolate mofetil, leflunomide and/or rituximab). Regarding other drugs, $42.8 \%$ of patients with DM and $42.8 \%$ of patients with ASS used an anti-hypertensive drug (beta blocker or calcium channel blocker) (Table 2).

Concerning comorbidities, 6 out of 14 DM patients, and 3 out of 7 ASS patients had systemic arterial hypertension. Dyslipidemia was identified in 9 out of 14 patients with DM, and 6 out of 7 patients with ASS.

No differences in the inter-observer reproducibility (healthy individuals) were observed between the two measurements of endothelial baseline diameter $(4.5 \pm$ $0.4 \mathrm{~mm}$ vs. $4.5 \pm 0.4 \mathrm{~mm}, P=0.920)$, hyperemia diameter $(4.5 \pm 0.3 \mathrm{~mm} v s .4 .5 \pm$ $0.4 \mathrm{~mm}, P=0.911)$ and FMD $(1.2 \% \pm 1.0 \% v s .1 .4 \% \pm 2.0 \%, P=0.919)$.

Endothelial baseline diameter $(P=0.130)$, hyperemia diameter $(P=0.282)$, and FMD values $(P=0.451)$ were similar among the ASS, DM and CTR groups. Moreover, FMD values were also similar in patients with DM and patients ASS $(P=0.585)$ (Figure 1$)$.

PWV values were also similar among patients in the DM, ASS and CTR groups $(P=0.253)$. In addition, there was no difference between patients with DM and patients with ASS ( $P \geq 0.999)$ (Figure 2).

\section{Discussion}

In the present study, we found no differences in the structural and functional characteristics of large arterial vessels in adult females with stable forms of DM and ASS and healthy individuals.

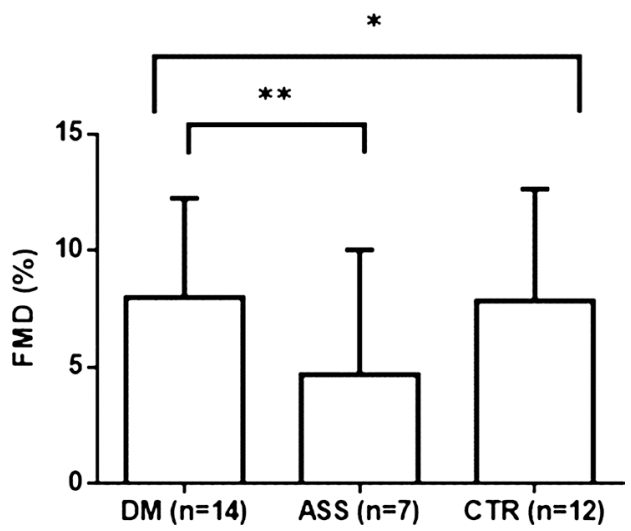

FMD: flow mediated dilatation; $\Delta$ : percentage changes in relation to baseline parameter. ${ }^{*}$ : Comparison among three groups (DM, ASS and CTR). $P=0.451{ }^{* *}$ : Comparison between DM and ASS patients. $P=0.585$.

Figure 1. Endothelial function before and after exercise. 


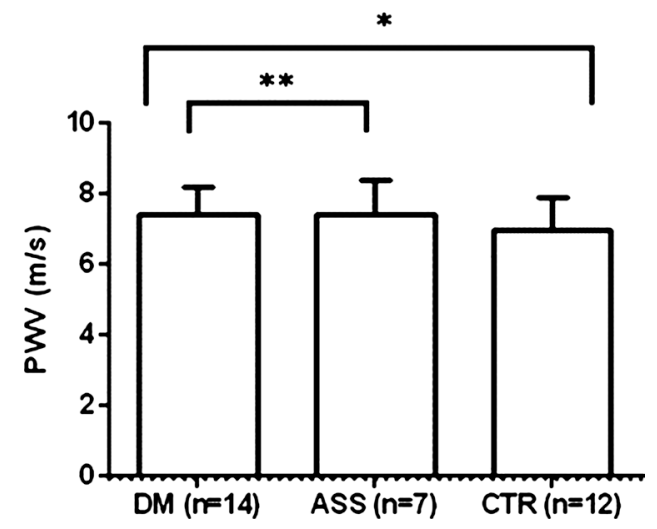

PWV: pulse wave velocity; $\Delta$ : percentage changes in relation to baseline parameter. ${ }^{*}$ : Comparison among three groups (DM, ASS and CTR). $P=0.253{ }^{* *}$ : Comparison between DM and ASS patients. $P=1.000$.

Figure 2. Structural properties of large arteries before and after exercise.

Our patients were submitted to strict exclusion criteria to avoid any confounding factors that could interfere with the interpretation of our results. For instance, we included only patients with stable diseases, described pharmacological treatment details, and excluded patients with diabetes mellitus and uncontrolled systemic arterial hypertension. Furthermore, the patients were age-, gender- and ethnicity-matched to members of the CTR group.

In this context, there were no differences between patients with $\mathrm{DM}$ and patients with ASS in relation to the CTR group. Likewise, in a sub-analysis the vascular parameters were comparable between patients with $\mathrm{DM}$ and patients with ASS.

Our results corroborate the only one available study in the literature [19] which showed no differences in the PWV and FMD of DM patient's parameters compared to a healthy control group. However, we specify patients' clinical, laboratory, pharmacological treatment, and disease status, and the patients matched by age, gender and ethnicity variables.

In this context, several studies have identified associations between disease status and the worsening of vascular structure and function [20] [21]. However, the chronic use of glucocorticoids results in increased risk factors for cardiovascular diseases, particularly obesity, diabetes and dyslipidemia [22] [23].

In an additional analysis, that considering only patients with DM, Vincze et al. [7] observed a reduction in the patients' FMD values when compared to those of healthy individuals. However, in this additional analysis, characteristics related to the disease status and the patients' general characteristics were not described, and the study did not mention whether the patients' general characteristics were matched to the CTR group [19]. In our study, we observed in a sub-analysis that the values of DM and ASS patients' FMD and PWV values were comparable to those of the CTR group, which was analyzed separately and matched by age, gender and ethnicity. This provided reliable results.

Our study's results suggest that stable SAMs with dyslipidemia or systemic arterial hypertension do not promote eventual endothelial dysfunction or in- 
creased arterial stiffness per se as previously reported in some studies [21].

Regarding our study's limitations, the number of patients in the present study precludes any population stratification. In addition, we did not analyze other variables related to endothelial function (i.e., menstrual cycle, retrograde and anterograde flow), so we cannot propose an overall understanding of the functional arterial parameters. Given these limitations, it is eminently necessary to carry out studies that examine such variables.

In conclusion, there are no differences in the structural and functional parameters of large arterial vessels in adult females with stable forms of DM and ASS, when compared to healthy individuals.

\section{Conflicts of Interest}

The authors declare no conflicts of interest regarding the publication of this paper.

\section{References}

[1] Dalakas, M.C. (2015) Inflammatory Muscle Diseases. The New England Journal of Medicine, 373, 393-394. https://doi.org/10.1056/NEJMc1506827

[2] Dalakas, M.C. (2015) Inflammatory Muscle Diseases. The New England Journal of Medicine, 372, 1734-1747. https://doi.org/10.1056/NEJMra1402225

[3] Connors, G.R., Christopher-Stine, L., Oddis, C.V. and Danoff, S.K. (2010) Interstitial Lung Disease Associated with the Idiopathic Inflammatory Myopathies: What Progress Has Been Made in the Past 35 Years? Chest, 138, 1464-1674. https://doi.org/10.1378/chest.10-0180

[4] Corretti, M.C., Anderson, T.J., Benjamin, E.J., Celermajer, D., Charbonneau, F., Creager, M.A., et al. (2002) Guidelines for the Ultrasound Assessment of Endothelial-Dependent Flow-Mediated Vasodilation of the Brachial Artery: A Report of the International Brachial Artery Reactivity Task Force. Journal of the American College of Cardiology, 39, 257-265. https://doi.org/10.1016/S0735-1097(01)01746-6

[5] Asmar, R., Benetos, A., Topouchian, J., Laurent, P., Pannier, B., Brisac, A.M., et al. (1995) Assessment of Arterial Distensibility by Automatic Pulse Wave Velocity Measurement. Validation and Clinical Application Studies. Hypertension, 26, 485-490. https://doi.org/10.1161/01.HYP.26.3.485

[6] Woodman, R.J., Playford, D.A., Watts, G.F., Cheetham, C., Reed, C., Taylor, R.R., et al. (2001) Improved Analysis of Brachial Artery Ultrasound Using a Novel Edge-Detection Software System. Journal of Applied Physiology, 91, 929-937. https://doi.org/10.1152/jappl.2001.91.2.929

[7] Silva, M.G., Borba, E.F., Mello, S.B. and Shinjo, S.K. (2016) Serum Adipocytokine Profile and Metabolic Syndrome in Young Adult Female Dermatomyositis Patients. Clinics (Sao Paulo), 71, 709-714. https://doi.org/10.6061/clinics/2016(12)06

[8] de Moraes, M.T., de Souza, F.H., de Barros, T.B. and Shinjo, S.K. (2013) Analysis of Metabolic Syndrome in Adult Dermatomyositis with a Focus on Cardiovascular Disease. Arthritis Care \& Research (Hoboken), 65, 793-799. https://doi.org/10.1002/acr.21879

[9] de Souza, F.H. and Shinjo, S.K. (2014) The High Prevalence of Metabolic Syndrome in Polymyositis. Clinical and Experimental Rheumatology, 32, 82-87. 
[10] Oliveira, D.S., Silva, M.G. and Shinjo, S.K. (2018) Insulin Resistance Is Increased in Adult Patients with Dermatomyositis. Medical Express, 5, mo18003. https://doi.org/10.5935/MedicalExpress.2018.mo.003

[11] Lundberg, I.E., Tjärnlund, A., Bottai, M., Werth, V.P., Pilkington, C., de Visser, M., et al. (2017) 2017 European League against Rheumatism/American College of Rheumatology Classification Criteria for Adult and Juvenile Idiopathic Inflammatory Myopathies and Their Major Subgroups. Arthritis \& Rheumatology, 69, 2271-2282. https://doi.org/10.1002/art.40320

[12] Miller, F.W., Rider, L.G., Chung, Y.L., Cooper, R., Danko, K., Farewell, V., et al. (2001) Proposed Preliminary Core Set Measures for Disease Outcome Assessment in Adult and Juvenile Idiopathic Inflammatory Myopathies. Rheumatology (OXford), 40, 1262-1273. https://doi.org/10.1093/rheumatology/40.11.1262

[13] Bruce, B. and Fries, J.F. (2003) The Stanford Health Assessment Questionnaire: Dimensions and Practical Applications. Health and Quality of Life Outcomes, 1, 20. https://doi.org/10.1186/1477-7525-1-20

[14] Rider, L.G., Giannini, E.H., Harris-Love, M., Joe, G., Isenberg, D., Pilkington, C., et al. (2003) Defining Clinical Improvement in Adult and Juvenile Myositis. The Journal of Rheumatology, 30, 603-617.

[15] Harris-Love, M.O., Shrader, J.A., Koziol, D., Pahlajani, N., Jain, M., Smith, M., et al. (2009) Distribution and Severity of Weakness among Patients with Polymyositis, Dermatomyositis and Juvenile Dermatomyositis. Rheumatology (Oxford), 48, 134-139. https://doi.org/10.1093/rheumatology/ken441

[16] Isenberg, D.A., Allen, E., Farewell, V., Ehrenstein, M.R., Hanna, M.G., Lundberg, I.E., et al. (2004) International Consensus Outcome Measures for Patients with Idiopathic Inflammatory Myopathies. Development and Initial Validation of Myositis Activity and Damage Indices in Patients with Adult Onset Disease. Rheumatology (Oxford), 43, 49-54. https://doi.org/10.1093/rheumatology/keg427

[17] Rider, L.G., Feldman, B.M., Perez, M.D., Rennebohm, R.M., Lindsley, C.B., Zemel, L.S., et al. (1997) Development of Validated Disease Activity and Damage Indices for the Juvenile Idiopathic Inflammatory Myopathies: I. Physician, Parent, and Patient Global Assessments. Juvenile Dermatomyositis Disease Activity Collaborative Study Group. Arthritis \& Rheumatology, 40, 1976-1983.

https://doi.org/10.1002/art.1780401109

[18] Piepoli, M.F., Hoes, A.W., Agewall, S., Albus, C., Brotons, C., Catapano, A.L., et al. (2016) 2016 European Guidelines on Cardiovascular Disease Prevention in Clinical Practice: The Sixth Joint Task Force of the European Society of Cardiology and Other Societies on Cardiovascular Disease Prevention in Clinical Practice (Constituted by Representatives of 10 Societies and by Invited Experts). Developed with the Special Contribution of the European Association for Cardiovascular Prevention \& Rehabilitation (EACPR). European Heart Journal, 37, 2315-2381.

https://doi.org/10.1093/eurheartj/ehw106

[19] Vincze, M., Dér, H., Kerekes, G., Szodoray, P., Zeher, M., Dankó, K., et al. (2014) Decreased Flow-Mediated Dilatation with Increased Arterial Stiffness and Thickness as Early Signs of Atherosclerosis in Polymyositis and Dermatomyositis Patients. Clinical Rheumatology, 33, 1635-1641.

https://doi.org/10.1007/s10067-014-2561-y

[20] Lima, D.S., Sato, E.I., Lima, V.C., Miranda, F. and Hatta, F.H. (2002) Brachial Endothelial Function Is Impaired in Patients with Systemic Lupus Erythematosus. The Journal of Rheumatology, 29, 292-297. 
[21] Soltesz, P., Kerekes, G., Der, H., Szucs, G., Szanto, S., Kiss, E., et al. (2011) Comparative Assessment of Vascular Function in Autoimmune Rheumatic Diseases: Considerations of Prevention and Treatment. Autoimmunity Reviews, 10, 416-425. https://doi.org/10.1016/j.autrev.2011.01.004

[22] Giles, J.T., Szklo, M., Post, W., Petri, M., Blumenthal, R.S., Lam, G., et al. (2009) Coronary Arterial Calcification in Rheumatoid Arthritis: Comparison with the Multi-Ethnic Study of Atherosclerosis. Arthritis Research \& Therapy, 11, R36. https://doi.org/10.1186/ar2641

[23] Giles, J.T., Danielides, S., Szklo, M., Post, W.S., Blumenthal, R.S., Petri, M., et al. (2015) Insulin Resistance in Rheumatoid Arthritis: Disease-Related Indicators and Associations with the Presence and Progression of Subclinical Atherosclerosis. Arthritis \& Rheumatology, 67, 626-636. https://doi.org/10.1002/art.38986 